\title{
Trastuzumab-based Retreatment after Lapatinib in Heavily Pretreated HER2 Positive Metastatic Breast Cancer: an Anatolian Society of Medical Oncology Study
}

\author{
Dogan Uncu ${ }^{1 *}$, Ibrahim Vedat Bayoglu², Ulku Yalcintas Arslan ${ }^{3}$, Mehmet \\ Kucukoner $^{4}$, Mehmet Artac ${ }^{5}$, Dogan Koca ${ }^{6}$, Arzu Oguz ${ }^{7}$, Umut Demirci ${ }^{3}$, Erkan \\ Arpaci $^{8}$, Mutlu Dogan ${ }^{1}$, Yuksel Kucukzeybek ${ }^{2}$, Ibrahim Turker ${ }^{3}$, Abdurrahman \\ Isikdogan $^{4}$, Tunc Guler ${ }^{5}$, Nurullah Zengin ${ }^{1}$
}

\begin{abstract}
Background: For HER2 positive metastatic breast cancer (MBC), continuing anti-HER2 therapy beyond progression is associated with improved outcome. However retreatment with trastuzumab after lapatinib progression is controversial. We retrospectively analyzed the efficacy of trastuzumab-based chemotherapy in HER2+ metastatic breast cancer patients whose disease progressed after lapatinib. Materials and Methods: Between October 2010 and May 2013, 54 patients whose disease progressed after lapatinib were retreated with trastuzumab-based chemotherapy. Efficacy and toxicity results were evaluated retrospectively. Results: The median age of patients was 46 (range 27-67). Fourteen patients (26\%) had metastases at the time of diagnosis. All of the patients had received trastuzumab in an adjuvant or metastatic setting, while $16(30 \%)$ had received two lines of trastuzumab. All patients had received lapatinib plus capecitabine. The median chemotherapy line for the metastatic setting was 2 (range 1-7). Cranial metastases were identified in 27 (50\%) patients. 53 patients received trastuzumab-based chemotherapy following lapatinib progression while one patient received trastuzumab monotherapy. Combination chemotherapy consisted of navelbin $(n=33)$, taxane $(n=10)$, gemcitabine $(n=2)$, platinum $(n=2)$ and platinum with taxane $(n=6)$. The median treatment cycle was 5 (range 1-44). Among 49 patients assessed for response 2 (4\%) showed CR, 12 (25\%) PR,11 (22\%) SD and 24 (49\%) disease progression. Asymptomatic cardiotoxicity was reported in 2 (4\%) of the patients. At a median follow-up of 9 months (1-39), median progression-free survival was 5 months $(95 \%$ CI 4.1-5.9) and median overall survival was 10 months (95\% CI 6.9-13.0). PFS and OS were not affected by the absence/presence of cranial metastases. Conclusions: Retreatment with trastuzumab-based therapy after lapatinib progression showed efficacy in heavily treated MBC patients.
\end{abstract}

Keywords: Breast cancer - metastatic - HER2 positive - trastuzumab - lapatinib - retreatment

Asian Pac J Cancer Prev, 16 (9), 4127-4131

\section{Introduction}

Breast carcinoma is not only the most common cancer but also the leading cause of cancer-related death in women in most countries all over the world (Siegel et al., 2013). Metastatic breast cancer (MBC) is an incurable disease. However, in the last decade new targeted agents and new combination therapies have been shown to lengthen patient survival and improve quality of life in MBC (Cabuk et al., 2014). HER2 overexpression occurs in $25 \%-30 \%$ of cases of $\mathrm{MBC}$ and is an adverse prognostic indicator associated with aggressive clinical course and consequently with a poor prognosis and shortened survival (Revillion et al., 1998; Chariyalertsak et al., 2011; Ghaffari et al., 2011; Singhai et al., 2011).

Randomized trials have shown that a combination of chemotherapy with trastuzumab increases response rates and prolongs survival in patients with HER2 positive MBC (Slamon et al., 2001; Marty et al., 2005). However most of the patients demonstrate disease progression after 1 year trastuzumab use (Sendur et al., 2012).

The benefit of continued use of anti-HER2 therapies beyond progression in HER2 positive MBC is associated with improved outcome but its optimal duration of administration is unknown. In two prospective randomized phase III studies, trastuzumab in combination with capecitabine or with lapatinib was more effective than single agent capecitabine or single agent lapatinib, 
respectively (von Minckwitz et al., 2009; Blackwell et al., 2010). In another trial, lapatinib in combination with capecitabine was superior to capecitabine in patients progressing following trastuzumab (Geyer et al., 2006). There is few knowledge about lapatinib resistance but differentially expressed genes may be responsable for the resistance (Zhuo et al., 2014).

Scaltriti et al. (2009) reported that lapatinib may resensitize HER2 positive cells to subsequent trastuzumab in a preclinical study. Little clinical data is available about the efficacy of retreatment with trastuzumab after lapatinib.

This multicenter retrospective study reports the clinical outcome of HER2 positive MBC patients who were re-challenged with trastuzumab-based therapy after progression following lapatinib and capecitabine.

\section{Materials and Methods}

Fifty-four HER2 positive MBC patients retreated with trastuzumab-based therapy after lapatinib progression between October 2010 and May 2013 were evaluated retrospectively. Patients data were recruited from eight centers of the Anatolian Society of Oncology Centers in Turkey.

The median age was 46 years and the range was 27-67 years. Fifty three patients were female and one patient was male. Twenty six (48\%) patients were ER or PR positive. HER 2 positivity was determined as immunohistochemical staining of $3+$ in $48(89 \%)$ patients or $2+$ with FISH amplified in $6(11 \%)$ patients.

Forty eight $(89 \%)$ of the patients had received anthracycline and 50 (93\%) of the patients had received taxane therapy previously. Fourteen (26\%) patients had metastases at the time of diagnosis.

All of the patients had received prior trastuzumab in an adjuvant or metastatic setting. Forty-seven (87\%) patients had received trastuzumab in a metastatic setting, while $16(30 \%)$ patients had received two lines of trastuzumab based chemotherapy. All of the patients had received lapatinib and capecitabine. Median chemotherapy lines for the metastatic setting were 2 and ranged from 1-7 lines, and 26 patients (48\%) had received at least 3 lines of chemotherapy.

Thirty nine $(72 \%)$ patients had at least 2 organ metastases and $27(50 \%)$ had cranial metastases. The characteristics of patients at the time of rechallenge with trastuzmab are summarized in Table 1.

\section{Statistical analysis}

Tumor response was analysed according to Response Evaluation Criteria in Solid Tumors guideline version 1.0 (Therasse et al., 2000). Overall response rate (ORR) was defined as the proportion of patients achieving complete or partial response among those with measurable disease. Toxicity was assessed according to the National Cancer Institute Common Toxicity Criteria version 2.0 (Trotti et al., 2000).

Progression free survival (PFS) was defined as the interval from the first day in which rechallenge with trastuzumab-based therapy started until tumor progression or last control, and overall survival (OS) was defined as the interval from the first day of this therapy to death or last control.

SPSS 13.0 for Windows package program was used for statistical analysis. Survival rates were calculated according to the Kaplan-Meier Method. Local ethical committee approval was obtained.

\section{Results}

All patients were pretreated with trastuzumab-based therapy and lapatinib and capecitabine. Trastuzumab retreatment was used as monotherapy in one patient, and in combination in 53 patients. In Table 2, the details of retreatment with trastuzumab-based therapy after lapatinib progression are summarized. Vinorelbine was the agent most frequently combined with trastuzumab $(n=33)$, while a combination of trastuzumab and taxanes was the most commonly used regimen. Six patients received platinum and taxane as triplet combination therapy.

Median treatment cycle was 5 (range 1-44). Forty-nine

\section{Table 1. Tumor and Patient Characteristics $(n=54)$}

\begin{tabular}{|c|c|c|}
\hline Characteristics & $\mathrm{n}$ & $(\%)$ \\
\hline Median age, years (range) & 46 & $(17-67)$ \\
\hline \multicolumn{3}{|l|}{ Sex } \\
\hline Male & 1 & 2 \\
\hline Female & 53 & 98 \\
\hline \multicolumn{3}{|l|}{ ECOG performance status } \\
\hline 0 & 8 & 15 \\
\hline 1 & 28 & 52 \\
\hline 2 & 14 & 26 \\
\hline 3 & 4 & 7 \\
\hline \multicolumn{3}{|l|}{ Hormone receptor status } \\
\hline ER or PR positive & 26 & 48 \\
\hline ER and PR negative & 28 & 52 \\
\hline \multicolumn{3}{|l|}{ Tumor Grade } \\
\hline G1 & 2 & 4 \\
\hline $\mathrm{G} 2$ & 16 & 30 \\
\hline G3 & 24 & 44 \\
\hline Unknown & 12 & 22 \\
\hline \multicolumn{3}{|l|}{ Stage at diagnosis } \\
\hline I & 2 & 4 \\
\hline II & 9 & 16 \\
\hline III & 29 & 54 \\
\hline IV & 14 & 26 \\
\hline \multicolumn{3}{|l|}{ No. of metastatic sites } \\
\hline 1 & 15 & 28 \\
\hline 2 & 19 & 35 \\
\hline$>3$ & 20 & 37 \\
\hline Cranial metastases & 27 & 50 \\
\hline \multicolumn{3}{|l|}{ Previous chemotherapy } \\
\hline Anthracycline & 48 & 89 \\
\hline Taxane & 50 & 93 \\
\hline Trastuzumab & 54 & 100 \\
\hline Lapatinib and capecitabine & 54 & 100 \\
\hline \multicolumn{3}{|l|}{ Previous trastuzumab } \\
\hline Adjuvant line & 14 & 26 \\
\hline Metastatic line & 47 & 87 \\
\hline \multicolumn{3}{|c|}{ Treatment of metastatic disease before trastuzumab retreatment } \\
\hline $\begin{array}{l}\text { Median number of prior chemotherapy } \\
\text { lines (range) }\end{array}$ & 2 & $(1-7)$ \\
\hline$>2$ line of treatment & 26 & 48 \\
\hline$>1$ line trastuzumab & 16 & 30 \\
\hline
\end{tabular}


patients could be assessed for response. Trastuzumabbased retreatment was associated with 2 (4\%) CR, 12 (25\%) PR and 11 (22\%) SD (Table 3). Objective response rate was $29 \%$.

At a median follow-up of 9 months (range 1-39), 45 patients had disease progression, and 34 patients had died. Median progression-free survival was 5 months $(95 \%$ CI 4.1-5.9) and median overall survival was 10 months (95\% CI 6.9-13.0) (Figure 1). Progression free survival and OS were not affected by the absence/presence of cranial metastases $(\mathrm{p}=0.6$ and $\mathrm{p}=0.3$ respectively).

Estrogen receptor status $(\mathrm{p}=0.34)$, progesteron receptor status $(\mathrm{p}=0.22)$, cranial metastasis $(\mathrm{p}=0.39)$, previous trastuzumab lines ( 1 vs 2 lines, $\mathrm{p}=0.38)$ and/ or previous number of treatment lines ( $<2$ lines $v s \geq 3$ lines, $\mathrm{p}=0.82$ ) had no statistically significant effect on OS in univariate analysis. Multivariate analysis could not be performed since none of these parameters had any significant effect on OS.

No unexpected toxicity was observed during retreatment with trastuzumab and most of the adverse events were mild. There was no treatment related death. Dose reduction was made in $12(25 \%)$ patients and cycle delay in 7 (13\%) patients.

Cardiotoxicity was reported in $2(4 \%)$ patients. These patients discontinued trastuzumab in combination due to grade 2 asymptomatic decrease in left ventricular ejection fraction (LVEF) below normal limits.

Severe adverse events were reported in $5(9 \%)$ patients. Two patients developed febril neutropenia. Two patients developed G3 neutropenia, and one patient developed G3 neutropenia and G3 anemia.

Table 2. Trastuzumab Combination Regimen after Lapatinib Progression (n=54)

\begin{tabular}{lrr}
\hline & $\mathrm{n}$ & $(\%)$ \\
\hline Chemotherapy combined with trastuzumab & & \\
Taxane & 10 & 18 \\
Gemcitabine & 2 & 4 \\
Vinorelbine & 33 & 61 \\
Platinum compound & 2 & 4 \\
Platinum compound with taxane & 6 & 11 \\
Trastuzumab monotherapy & 1 & 2 \\
\hline
\end{tabular}

Table 3. Tumor Response and Clinical Outcome of Patients

\begin{tabular}{lrc}
\hline & $\mathrm{n}$ & $(\%)$ \\
\hline Patients assessable for response & 49 & 91 \\
Objective response & & \\
ORR & 14 & 29 \\
CR & 2 & 4 \\
PR & 12 & 25 \\
SD & 11 & 22 \\
PD & 24 & 49 \\
Median follow up, months (range) & 9 & $(1-39)$ \\
Median progression-free survival, & 5 & $(4.1-5.9)$ \\
months (95\% CI) & & \\
Median overall survival, months (95\% CI) & 10 & $(6.9-13.0)$ \\
*CR, complete response; PR, partial response; SD, stable disease; PD, \\
progressive disease; ORR, overall response rate &
\end{tabular}
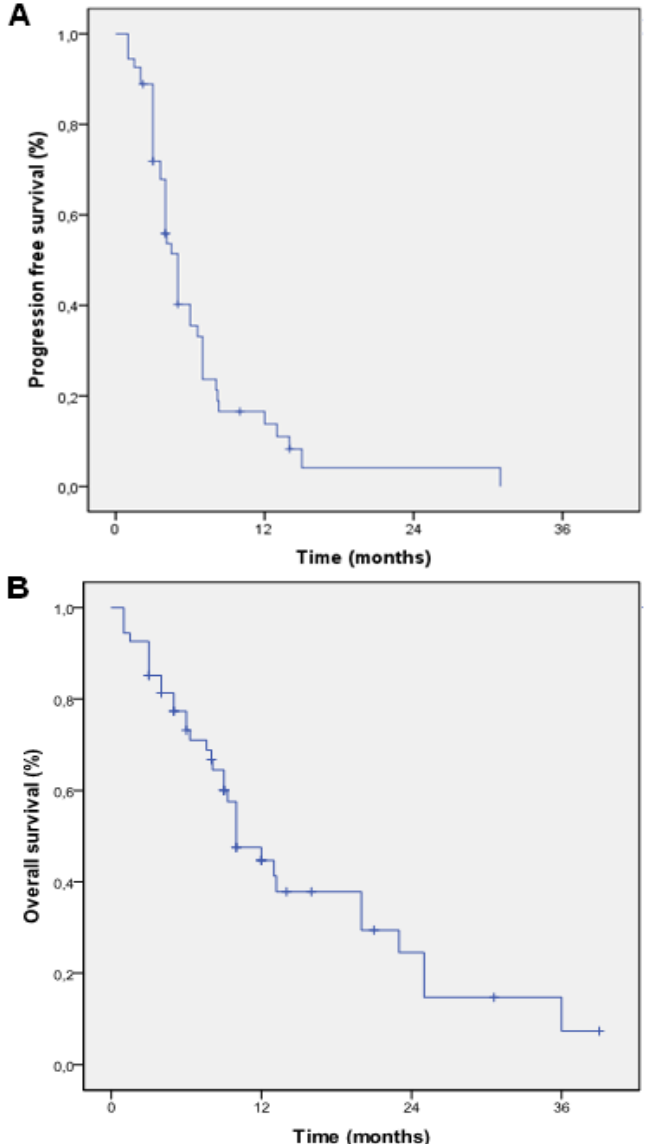

Figure 1. Kaplan-Meier curve of of PFS (A) and OS (B)

\section{Discussion}

This retrospective analysis evaluated the clinical outcome of 54 HER2 positive heavily treated MBC patients from 8 medical oncology departments in Turkey retreated with trastuzumab-based chemotherapy after lapatinib progression. In 49 patients who could be assessed for response, 29\% ORR was observed. Median PFS was 5 months and median OS was 10 months. Treatment was well tolerated and most of the adverse events were mild. No unexpected toxicity was observed during retreatment with trastuzumab. Grade 2 cardiotoxicity was reported in $2(4 \%)$ patients and severe adverse events were reported in $5(9 \%)$ patients, which in all cases were haematologic toxicities.

Prospective studies (Stemmler et al., 2005; Cancello et al., 2008; Extra et al., 2010) have shown that continuation of anti-HER2 therapies in patients progressing after trastuzumab-based treatment was associated with improved response rates and improved survival in HER2positive MBC patients. Also in prospective randomized phase III studies (von Minckwitz et al., 2009; Blackwell et al., 2010), trastuzumab combination with capecitabine or lapatinib resulted in improved progression free survival compared to single agent capecitabine or lapatinib.

Lapatinib and capecitabine (Geyer et al., 2006) is another option for patients progressing after trastuzumab. But after this treatment there are controversies concerning the efficacy of trastuzumab retreatment. A post-hoc analysis of EGF100151 study (Crown et al., 2009), suggests that subsequent anti-HER2 therapy is a valid 
Doğan Uncu et al

option following lapatinib + capecitabine. Although the analysis was retrospective in this study, and conducted on subgroups defined by posttreatment variables, the OS difference observed between patients receiving subsequent anti-HER2 therapy $v s$ those who did not may suggest that continued HER2 suppression is clinically relevant.

To date there are two retrospective series concerning about retreatment with trastuzumab after lapatinib progression. Spazzapan et al. (2010), reported 21 HER2 positive MBC patients retreated with trastuzumab with a $48 \%$ ORR, a median PFS of 6 months and median OS of 21.4 months. Recently, Gori et al. (2012), reported 69 patients who had received HER2 targeting agents for a median of two years. In this study, patients retreated with trastuzumab based chemotherapy after lapatinib progression showed a $31 \%$ ORR, with a median PFS of 5 months and median OS of 16 months.

In our series, the efficacy results of retreatment with trastuzumab after lapatinib progression confirmed these trials. Continuation of trastuzumab after lapatinib and capecitabine therapy is associated with a $29 \%$ ORR and median 5 months of PFS in heavily pretreated HER2 positive MBC patients. Our study group had worse prognostic parameters than the previous studies. ECOG performance status was $\geq 2$ in $33 \%$ of patients, $44 \%$ had grade 3 tumor and $50 \%$ had cranial metastases. Perhaps because of these worse prognostic parameters the median OS was shorter than the reported trials.

HER2 positive MBC patients are more likely to develop cranial metastases (Pestalozzi et al., 2006). However, patients with HER2 positive disease with cranial metastases treated with trastuzumab based chemotherapy have a better survival outcome than patients with HER2 negative disease and cranial metastases (Kirsch et al., 2005; Sawrie et al., 2007).

It is suggested that patients with cranial metastases should receive trastuzumab with chemotherapy for the cranial metastases (Bartsch et al., 2007). In our series PFS and OS were not affected by the absence/presence of cranial metastases.

The number of HER2 targeted therapies continues to grow and pertuzumab and the antibody-conjugate trastuzumab-DM1 are now available for treating HER2 positive metastatic breast cancer. Pertuzumab was evaluated in combination with trastuzumab (Swain et al., 2013) and an objective response rate of $80.2 \%$ was reported. In the EMILIA study (Verma et al., 2012), in patients treated with T-DM1 progressing after trastuzumab, an ORR of $43.6 \%$ and median PFS of 9.6 months were observed. In a post hoc exploratory analysis (Burris et al., 2011), T-DM1 was administered in a subgroup of patients pretreated with trastuzumab and lapatinib and an ORR of $24 \%$ was reported, similar to our results $(29 \%)$.

In conclusion, retreatment with trastuzumab-based therapy after lapatinib progression showed efficacy in heavily treated HER 2 positive MBC patients. Continuing HER 2 blockade provides clinical benefit for patients who have received several anti-HER2 treatments.

\section{References}

Bartsch R, Rottenfusser A, Wenzel C, et al (2007). Trastuzumab prolongs overall survival in patients with brain metastases from Her2 positive breast cancer. J Neurooncol, 85, 311-7.

Blackwell KL, Burstein HJ, Storniolo AM, et al (2010). Randomized study of Lapatinib alone or in combination with trastuzumab in women with ErbB2-positive, trastuzumabrefractory metastatic breast cancer. J Clin Oncol, 28, 1124-0.

Burris HA, Rugo HS, Vukelja SJ, et al (2011). Phase II study of the antibody drug conjugate trastuzumab-DM1 for the treatment of human epidermal growth factor receptor 2 (HER2)-positive breast cancer after prior HER2-directed therapy. J Clin Oncol, 29, 398-405.

Cabuk D, Basaran G, Teomete M, et al (2014). Clinical outcome of Turkish metastatic breast cancer patients with currently available treatment modalities-single center experience. Asian Pac J Cancer Prev, 15, 117-22.

Cancello G, Montagna E, D'Agostino D, et al (2008). Continuing trastuzumab beyond disease progression: outcomes analysis in patients with metastatic breast cancer. Breast Cancer Res, 10, 60.

Chariyalertsak S, Purisa W, Vinyuvat S (2011). Her-2/neu amplification determined by real-time quantitative PCR and its association with clinical outcome of breast cancer in Thailand. Asian Pac J Cancer Prev, 12, 1703-6.

Crown J, Casey M A, Cameron D, et al (2009). Lapatinib (L) plus capecitabine (C) in HER2+ metastatic breast cancer (MBC): exploratory analyses by prior therapy. Eur J Cancer , 7, 5082 .

Extra JM, Antoine EC, Vincent-Salomon A, et al (2010). Efficacy of trastuzumab in routine clinical practice and after progression for metastatic breast cancer patients: the observational Hermine study. Oncologist, 15, 799-809.

Geyer CE, Forster J, Lindquist D, et al (2006). Lapatinib plus capecitabine for HER2-positive advanced breast cancer. $N$ Engl J Med, 355, 2733-43.

Ghaffari SR, Sabokbar T, Dastan J, et al (2011). Her2 amplification status in Iranian breast cancer patients: comparison of immunohistochemistry (IHC) and fluorescence in situ hybridisation (FISH). Asian Pac J Cancer Prev, 12, 1031-4.

Gori S, Montemurro F, Spazzapan S, et al (2012). Retreatment with trastuzumab-based therapy after disease progression following lapatinib in HER2-positive metastatic breast cancer. Ann Oncol, 23, 1436-41.

Kirsch DG, Ledezma CJ, Mathews CS, et al (2005). Survival after brain metastases from breast cancer in the trastuzumab era. J Clin Oncol, 23, 2114-6; author reply 6-7.

Marty M, Cognetti F, Maraninchi D, et al (2005). Randomized phase II trial of the efficacy and safety of trastuzumab combined with docetaxel in patients with human epidermal growth factor receptor 2-positive metastatic breast cancer administered as first-line treatment: the M77001 study group. J Clin Oncol, 23, 4265-74.

Pestalozzi BC, Zahrieh D, Price KN, et al (2006). Identifying breast cancer patients at risk for Central Nervous System (CNS) metastases in trials of the International Breast Cancer Study Group (IBCSG). Ann Oncol, 17, 935-44.

Revillion F, Bonneterre J, Peyrat JP (1998). ERBB2 oncogene in human breast cancer and its clinical significance. Eur $J$ Cancer, 34, 791-808.

Sawrie SM, Meredith RF, Spencer RA, et al (2007). "HER2neu status as a predictor of survival in patients with brain metastases from primary breast adenocarcinoma." Journal of Clinical Oncology, 2007 ASCO Annual Meeting Proceedings (Post-Meeting Edition). Vol 25, No 18S (June 20 Supplement): 1016.

Scaltriti M, Verma C, Guzman M, et al (2009). Lapatinib, a HER2 tyrosine kinase inhibitor, induces stabilization and accumulation of HER 2 and potentiates trastuzumab- 

dependent cell cytotoxicity. Oncogene, 28, 803-14.

Sendur MAN, Aksoy S, Ozdemir NY, et al (2012). What is the mechanism of progression with trastuzumab treatment-escape or resistance? Asian Pac J Cancer Prev, 13, 5915-6.

Siegel R, Naishadham D, Jemal A (2013). Cancer statistics, 2013. CA Cancer J Clin, 63, 11-30.

Singhai R, Patil V, Patil A (2011). Immunohistochemical (IHC) HER-2/neu and fluorescent-in situ hybridization (FISH) gene amplification of breast cancer in Indian women. Asian Pac J Cancer Prev, 12, 179-83.

Slamon DJ, Leyland-Jones B, Shak S, et al (2001). Use of chemotherapy plus a monoclonal antibody against HER2 for metastatic breast cancer that overexpresses HER2. $N$ Engl J Med, 344, 783-92.

Spazzapan S, Crivellari D, Tumolo S, et al (2010). Retreatment with trastuzumab after progression on lapatinib-based therapy in heavily pretreated HER2-positive metastatic breast cancer: a monoinstitutional experience. J Clin Oncol, 28, 15S Abstr 1082.

Stemmler HJ, Kahlert S, Siekiera W, et al (2005). Prolonged survival of patients receiving trastuzumab beyond disease progression for HER2 overexpressing metastatic breast cancer (MBC). Onkologie, 28, 582-6.

Swain SM, Kim SB, Cortes J, et al (2013). Pertuzumab, trastuzumab, and docetaxel for HER2-positive metastatic breast cancer (CLEOPATRA study): overall survival results from a randomised, double-blind, placebo-controlled, phase 3 study. Lancet Oncol, 14, 461-71.

Therasse P, Arbuck SG, Eisenhauer EA, et al (2000). New guidelines to evaluate the response to treatment in solid tumors. European organization for research and treatment of cancer, national cancer institute of the United States, national cancer institute of Canada. J Natl Cancer Inst, 92, 205-16.

Trotti A, Byhardt R, Stetz J, et al (2000). Common toxicity criteria: version 2.0. an improved reference for grading the acute effects of cancer treatment: impact on radiotherapy. Int J Radiat Oncol Biol Phys, 47, 13-47.

Verma S, Miles D, Gianni L, et al (2012). Trastuzumab emtansine for HER2-positive advanced breast cancer. $N$ Engl J Med, 367, 1783-91.

von Minckwitz G, du Bois A, Schmidt M, et al (2009). Trastuzumab beyond progression in human epidermal growth factor receptor 2-positive advanced breast cancer: a german breast group 26/breast international group 03-05 study. J Clin Oncol, 27, 1999-2006.

Zhuo WL, Zhang L, Xie QC, et al (2014). Identifying differentially expressed genes and screening small molecule drugs for lapatinib-resistance of breast cancer by a bioinformatics strategy. Asian Pac J Cancer Prev, 15, 10847-53. 